\title{
Tendencia de las cuentas por cobrar y ratios de actividad en la empresa Alpe Corporación S.A. en los periodos $2011-2015$
}

\section{Trends in accounts receivable and ratios of activity in the company Alpe Corporación S.A. in the periods $2011-2015$}

Umasi Olarte, Betzabeth; Choquepuma Olarte, Virginia

\author{
EP. Contabilidad, Facultad de Ciencias Empresariales, Universidad Peruana Unión
}

\begin{abstract}
Resumen
El trabajo de investigación tiene como objetivo determinar la tendencia de las cuentas por cobrar y ratios de actividad en la empresa Alpe Corporación S.A. en los periodos 2011 a junio 2015. El nivel de estudio es descriptivo, predictivo, longitudinal y retrospectivo. La población de estudio está conformada por la cartera de clientes de la empresa, de los cuales se recolectó los datos para determinar y pronosticar la tendencia de las cuentas por cobrar y analizar los ratios de actividad para determinar la frecuencia de recuperación de cuentas por cobrar y la rotación trimestral de la empresa Alpe Corporación S.A. durante el periodo 2011 al 2017, teniendo como datos históricos disponibles del 2011 a junio de 2015. Para el análisis estadístico se utilizó el paquete SPSS 20. Así mismo, se muestra el análisis del modelo ANOVA y el nivel de significancia y correlación entre la rotación de cuentas por cobrar y rotación trimestral del periodo 2011 al 2017 de la empresa, siendo la relación entre las variables es alta en el coeficiente de correlación $\mathrm{r}=, 986$ y r2 = ,973. Es decir, la variación de la rotación trimestral explicada por el modelo de regresión lineal, por lo cual, se puede predecir la rotación trimestral a partir de la Rotación de cuentas por cobrar al 98.6\%. A esto el valor P o sig = , $000<, 05(\alpha)$ indica que el modelo propuesto de regresión es significativo. Luego, teniendo en cuenta que el nivel óptimo de rotación de las cuentas por cobrar es de 6 a 12 veces al año y de 60 a 30 días de periodo de cobro; para fines de este estudio se considera un periodo desde el año 2011 al segundo trimestre del 2015 de acuerdo a los datos históricos disponibles. Es así que el nivel óptimo de rotación de cuentas por cobrar es de 1.5 a 3 veces por trimestre y de 60 a 30 días de periodo de cobro normal. Los resultados manifiestan que la tendencia de las cuentas por cobrar y los ratios de actividad, evidencia la eficiencia de la empresa Alpe Corporación S.A. en el periodo 2011 al 2017. Por lo cual, la empresa convierte en efectivo sus cuentas por cobrar en la medida que los días aumentan y las veces de cobro disminuye en cada trimestre de cada año. Lo que significa que la rotación de cuentas por cobrar, así como la rotación trimestral, se encuentra en el nivel óptimo, concluyendo que la capacidad gerencial de la empresa es eficiente para generar fondos de manera efectiva administrando de forma adecuada los servicios prestados proyectados a los trimestres que falten de julio de 2015, 2016 y 2017.
\end{abstract}

Palabras Clave: Cuentas por cobrar. Ratios de actividad.

\begin{abstract}
The main of the research work is to determine the trend of accounts receivable and activity ratios in the company Alpe Corporación S.A. in the periods 2011 to June 2015. The level of study is descriptive, predictive, longitudinal and retrospective. The study population is made up of the company's client portfolio, from which the data was collected to determine and forecast the trend of accounts receivable and analyze the activity ratios to determine the frequency of recovery of accounts receivable and the quarterly rotation of the company Alpe Corporación SA during the period 2011 to 2017, taking as historical data available from 2011 to June 2015. For the statistical analysis the SPSS 20 package was used. Also, the analysis of the ANOVA model and the level of significance and correlation between the rotation are shown. of accounts receivable and quarterly rotation of the period 2011 to 2017 of the company, being the relationship between the variables is high in the correlation coefficient $\mathrm{r}=, 986$ and $\mathrm{r} 2=$, 973. That is, the variation of the quarterly rotation explained by the linear regression model, therefore, the quarterly rotation can be predicted from the Rotation of accounts receivable to $98.6 \%$. To this the $\mathrm{P}$ value or sig $=, 000<, 05(\alpha)$ indicates that the proposed regression model is significant. Then, taking into account that the optimal level of turnover of accounts receivable is 6 to 12 times a year and 60 to 30 days of collection period; For the purposes of this study, a period from 2011 to the second quarter of 2015 is considered according to the available historical data. Thus, the optimum level of turnover of accounts receivable is 1.5 to 3 times per quarter and 60 to 30 days of normal collection period. The results show that the trend of accounts receivable and activity ratios, shows the efficiency of the company Alpe Corporación S.A. in the period 2011 to 2017. Therefore, the company converts accounts receivable into cash as days increase and collection times decrease in each quarter of each year. This means that the rotation of accounts receivable, as well as quarterly turnover, is at the optimum level, concluding that the managerial capacity of the company is efficient to generate funds in an effective manner, administering adequately the services provided projected to the missing trimesters of July 2015, 2016 and 2017.
\end{abstract}

Keywords: Accounts receivable. Activity ratios.

Autor de correspondencia:

Tel.: 936030971-959109508 


\section{Introducción}

Aguilar (2013) menciona que, en la actualidad las empresas emprenden grandes búsquedas de herramientas que permitan mejorar el desarrollo de sus actividades, todo ello en respuesta a los continuos cambios que se producen en el entorno y que las obligan a tomar medidas que permitan mantener el negocio en marcha, satisfaciendo las necesidades de su cartera de clientes y generando altos niveles de rentabilidad.

Noriega (2011) a fin de conservar los clientes actuales y atraer nuevos, las empresas deben conceder créditos y mantener inventarios. Las cuentas por cobrar representan el total del crédito otorgado por una empresa a sus clientes, el cual se determina a través de su volumen de ventas a crédito.

Esta modalidad será efectiva en la medida que esté debidamente programada y establecida, la fecha de vencimiento de la factura documento mismo que representa el compromiso que los clientes poseen con la empresa que le prestó el servicio, es decir, es necesario que las organizaciones tengan correctamente estipuladas las pautas y condiciones bajo las cuales se está ofreciendo esta modalidad de pago, de lo contrario es difícil conocer si las cuentas por cobrar se están haciendo efectivas en períodos de tiempos razonables.

En este sentido, la importancia de este estudio es relevante debido a que las cuentas por cobrar presentan beneficios, pero también provocan riesgos, por ello, se debe analizar las cuentas por cobrar según la rotación de actividad mediante los resultados trimestrales en el periodo 2011 a junio del 2015. Los cuales permiten pronosticar e describir las carteras atrasadas, pesadas y carteras de alto riesgo, así como, obtener informes de manera oportuna y precisa. Lo mismo que beneficiará y será de utilidad a las empresas privadas que otorguen créditos a distintos clientes.

De los resultados obtenidos, se espera que la empresa, de ser necesario tome medidas de mejora continua de gestión de cobranza, así como agilizar la toma de decisiones que ayuden a fortalecer el desarrollo y cumplir con los objetivos de la empresa, asimismo el personal involucrado del área de créditos y cobranzas incremente su capacidad para la recuperación de las cuentas por cobrar, permitiendo obtener los resultados que la administración, espera por ende, es importante y necesario proponer mejoras de las cuentas por cobrar, para hacer eficiente y así poder disminuir la rotación de este rubro.

El trabajo de investigación pretende determinar la tendencia de las cuentas por cobrar y ratios de actividad en la empresa Alpe Corporación en el periodo 2011 a junio 2015. Para tal efecto se analizaron los temas: las carteras atrasadas, carteras pesadas y cartera de alto riesgo en la empresa, y medir la eficiencia en la rotación de las cuentas por cobrar y el período de cobros o rotación trimestral, los cuales permiten determinar el comportamiento de la situación financiero en cinco (5) últimos años en la empresa.

Por lo cual, todo esto conlleva a formular las preguntas de investigación de acuerdo a la descripción del problema surge la investigación a desarrollarse, análisis de las cuentas por cobrar y la rotación de actividad en la empresa Alpe Corporación, que hace referencia al servicio y venta otorgados al crédito, dinero que será recuperado en un tiempo definido, dependiendo al contrato que se determinó con la empresa.

Según Aguilar (2013) definió que las cuentas por cobrar representan el total del crédito otorgado por una empresa a sus clientes; estas cuentas representan derechos exigibles originados por ventas, que luego pueden hacerse efectivos para la empresa que lo otorga, un medio de dinero a futuro porque origina el cobro de sus cuentas en un periodo posterior a su venta.

Asimismo, las cuentas por cobrar representan al dinero adeudado por las entidades, así como son derechos que le corresponde a la empresa que ha brindado un servicio o ha realizado una venta al crédito, dinero que será recuperado en un tiempo determinado, dependiendo al contrato que se determinó con la empresa.

Respecto a los ratios de actividad, Aching (2006) mencionó que mide la efectividad y eficiencia de la gestión. En la administración del capital de trabajo, expresan los efectos de decisiones y políticas seguidas por la empresa, con respecto a la utilización de sus fondos. Evidencian cómo se manejó la empresa en lo referente a cobranzas, ventas al contado, inventarios y ventas totales. Estos ratios implican una comparación entre ventas y activos necesarios para soportar el nivel de ventas, considerando que existe un apropiado valor de correspondencia entre estos conceptos.

Expresan la rapidez con que las cuentas por cobrar o los inventarios se convierten en efectivo. Son un complemento de las razones de liquidez, ya que permiten precisar aproximadamente el período de tiempo que la cuenta respectiva (cuenta por cobrar, inventario), necesita para convertirse en dinero. Miden la capacidad que tiene la gerencia para generar fondos internos, al administrar en forma adecuada los recursos invertidos en estos activos.

\section{Método}

La investigación se enmarca dentro del enfoque cuantitativo, no experimental. Cuantitativo porque pretende medir y describir las cuentas por cobrar y el ratio de actividad. No experimental porque no pretende manipular deliberadamente variables se basa fundamentalmente en la observación de fenómenos tal y como se dan en su contexto natural para después analizarlos. Es descriptiva por que pretende describir mediante una revisión y análisis de las cuentas por cobrar y el ratio de actividad en la empresa Alpe Corporación S.A. Asimismo, se soporta principalmente en técnicas como la observación y la revisión de registros 
históricos, cuyo objetivo es el logro de un análisis, evaluación, identificación, e interpretación de los hechos reales que ocurrirán a lo largo del estudio según (Carrasco, 2014). Por otro lado, se le considera una investigación predictiva que tiene como propósito prever o anticipar situaciones futuras, requiere la descripción, la comparación, el análisis y la explicación, esta investigación tipo pronóstico es aquella en cual el propósito principal es predecir la dirección futura de los eventos investigados (Gonzalez, 2013).

En este estudio retrospectivo, se consideró como unidad de análisis, de la data del sistema de cartera de clientes de la empresa Alpe Corporación S.A. en el periodo 2011 a junio del 2015.

En cuanto al análisis estadístico, se hizo uso de tablas de frecuencias, tablas porcentuales y tablas de índices. Así como también la estadística paramétrica como el análisis de regresión lineal simple coeficiente y determinación para pronosticar la tendencia de las cuentas por cobrar y determinar su eficacia en la empresa utilizando el paquete estadístico SPSS 20. La población de estudio está conformada por la cartera de clientes de la empresa Alpe Corporación S.A. en los periodos 2011-2015. Se recopiló información financiera de la data en la empresa Alpe Corporación S.A. el cual se utilizó para analizar la información de los 5 últimos años.

\section{Resultados}

Siendo que la empresa Alpe Corporación S.A. dedicada a las ventas y prestación de servicios a crédito se han convertido en los últimos años en un medio de ingreso significativo; es necesario determinar la tendencia de las cuentas por cobrar y ratios de actividad en la empresa Alpe Corporación S.A. de enero del año 2011 a junio del año 2015, para tal efecto se analizaron los temas: describir las carteras atrasadas, carteras pesadas y cartera de alto riesgo y medir la eficiencia en la rotación de las cuentas por cobrar y el período de cobros o rotación trimestral, los cuales permitirá determinar el comportamiento de la situación financiero en cinco (5) últimos años de la empresa.

La tabla 3 presenta el resumen de las carteras de clientes de los años 2011 a junio 2015. Lo cual se menciona; en el año 2011 en los cuatro trimestres, las cuentas por cobrar según el tipo de cartera; la cobranza vencida de la cartera atrasada y la cobranza con problemas potenciales registra la mayor cantidad de comprobantes con retraso, sin embargo la cobranza inicial asciende a importes altos; la cartera de alto riesgo en el cuarto trimestre, la cantidad de comprobantes asciende a un $25 \%$, en tal sentido el sistema de cobranzas de la empresa debería haber mejorado la estrategia de gestión al otorgar los créditos. Sin embargo, en los trimestres de los años 2012, 2013, 2014 y el primer semestre del 2015, la cobranza inicial correspondiente a la cartera atras ada representada en su mayoría, lo cual indica que la nueva política de gestión de cobranzas en la empresa ha mejorado considerablemente. En este sentido, es necesario aplicar políticas, procedimientos y períodos de crédito adecuados al giro del negocio, que proyecte beneficios económicos a las empresas.

Tabla 3

Resumen de comprobantes de pago de operaciones de ventas al crédito canceladas, según tipo de cartera, Periodo 2011 - junio 2015

RESUMEN DE CUENTAS POR COBRAR - PERIODO 2011 - JUNIO 2015

\begin{tabular}{|c|c|c|c|c|c|c|c|c|c|c|c|c|c|c|c|c|c|c|c|c|c|}
\hline \multirow{3}{*}{$\mathrm{N}^{\circ}$} & \multirow{3}{*}{ Año } & \multicolumn{8}{|c|}{ Cartera atrasada } & \multicolumn{4}{|c|}{ Carteras Pesadas } & \multicolumn{4}{|c|}{ Carteras de alto riesgo } & \multirow{2}{*}{\multicolumn{4}{|c|}{ Total }} \\
\hline & & \multicolumn{4}{|c|}{ Cobranza Inicial (normal) } & \multicolumn{4}{|c|}{ Cobranza vencida } & \multicolumn{4}{|c|}{ Con problemas potenciales. } & \multicolumn{4}{|c|}{ Castigo de cuentas incobrables } & & & & \\
\hline & & $\begin{array}{c}\text { Cantidad } \\
\text { Factura } \\
\text { Emitida }\end{array}$ & $\%$ & Monto Facturado & $\%$ & $\begin{array}{l}\text { Cantidad } \\
\text { Factura } \\
\text { Emitida }\end{array}$ & $\%$ & Monto Facturado & $\%$ & $\begin{array}{l}\text { Cantidad } \\
\text { Factura } \\
\text { Emitida }\end{array}$ & $\%$ & Monto Facturado & $\%$ & $\begin{array}{l}\text { Cantidad } \\
\text { Factura } \\
\text { Emitida }\end{array}$ & $\%$ & $\begin{array}{c}\text { Monto } \\
\text { Facturado }\end{array}$ & $\%$ & $\begin{array}{l}\text { Cantidad } \\
\text { Factura } \\
\text { Emitida }\end{array}$ & $\%$ & Monto Facturado & $\%$ \\
\hline 1 & 2011-1 & 98 & $18 \%$ & S/. 311,185 & $43 \%$ & 253 & $46 \%$ & S/. 299,604 & $41 \%$ & 196 & $35 \%$ & S/. 115,880 & $16 \%$ & 7 & $1 \%$ & S/. 2,440 & $0.3 \%$ & 554 & $6.3 \%$ & S/. 729,109 & $2.9 \%$ \\
\hline 2 & 2011-2 & 92 & $27 \%$ & S/. 333,046 & $57 \%$ & 187 & $55 \%$ & S/. 180,354 & $31 \%$ & 63 & $18 \%$ & S/. 67,582 & $12 \%$ & 0 & $0 \%$ & S/. 0 & $0 \%$ & 342 & $3.9 \%$ & S/. 580,981 & $2.3 \%$ \\
\hline 3 & 2011-3 & 141 & $29 \%$ & S/. 199,417 & $36 \%$ & 217 & $45 \%$ & S/. 192,843 & $35 \%$ & 122 & $25 \%$ & S/. 162,215 & $29 \%$ & 1 & $0.2 \%$ & S/. 19 & $0 \%$ & 481 & $5.5 \%$ & S/. 554,494 & $2.2 \%$ \\
\hline 4 & 2011-4 & 165 & $32 \%$ & S/. 243,350 & $44 \%$ & 118 & $23 \%$ & S/. 198,816 & $36 \%$ & 103 & $20 \%$ & S/. 57,739 & $11 \%$ & 128 & $25 \%$ & S/ 48,307 & $9 \%$ & 514 & $5.9 \%$ & S/. 548,212 & $2.2 \%$ \\
\hline 5 & $2012-1$ & 281 & $63 \%$ & S/. 576,818 & $58 \%$ & 78 & $18 \%$ & S/. 259,577 & $26 \%$ & 60 & $14 \%$ & S/. 140,828 & $14 \%$ & 25 & $6 \%$ & S/. 12,891 & $1 \%$ & 444 & $5.1 \%$ & S/. 990,114 & $3.9 \%$ \\
\hline 6 & 2012-2 & 272 & $66 \%$ & S/. 364,987 & $53 \%$ & 86 & $21 \%$ & S/. 224,311 & $32 \%$ & 48 & $12 \%$ & S/. 90,653 & $13 \%$ & 4 & $1 \%$ & S/. 14,782 & $2 \%$ & 410 & $4.7 \%$ & S/. 694,734 & $2.7 \%$ \\
\hline 7 & 2012-3 & 211 & $62 \%$ & S/. 561,553 & $50 \%$ & 75 & $22 \%$ & S/. 275,340 & $24 \%$ & 52 & $15 \%$ & S/. 284,654 & $25 \%$ & 5 & $1 \%$ & S/. 12,681 & $1 \%$ & 343 & $3.9 \%$ & S/. 1,134,228 & $4.5 \%$ \\
\hline 8 & $2012-4$ & 256 & $59 \%$ & S/. 1,478,864 & $80 \%$ & 60 & $14 \%$ & S/. 147,263 & $8 \%$ & 110 & $25 \%$ & S/. 222,128 & $12 \%$ & 7 & $2 \%$ & S/. 9,065 & $0.5 \%$ & 433 & $4.9 \%$ & S/. 1,857,319 & $7.3 \%$ \\
\hline 9 & 2013-1 & 214 & $39 \%$ & S/. 926,639 & $53 \%$ & 93 & $17 \%$ & S/. 469,279 & $27 \%$ & 235 & $43 \%$ & S/. 339,758 & $20 \%$ & 4 & $0.7 \%$ & S/. 3,810 & $0.2 \%$ & 546 & $6.2 \%$ & S/ 1, $\mathbf{1 7 3 9 , 4 8 5}$ & $6.8 \%$ \\
\hline 10 & 2013-2 & 221 & $49 \%$ & S/. 466,878 & $30 \%$ & 112 & $25 \%$ & S/. 381,987 & $25 \%$ & 118 & $26 \%$ & S/. 688,815 & $44 \%$ & 2 & $0.4 \%$ & S/. 11,458 & $0.7 \%$ & 453 & $5.2 \%$ & S/. 1,549,138 & $6.1 \%$ \\
\hline 11 & 2013-3 & 248 & $40 \%$ & S/. 738,249 & $41 \%$ & 98 & $16 \%$ & S/. 517,709 & $29 \%$ & 277 & $44 \%$ & S/. 529,829 & $30 \%$ & 1 & $0.2 \%$ & S/. 5,565 & $0.3 \%$ & 624 & $7.1 \%$ & S/. 1,791,352 & $7.0 \%$ \\
\hline 12 & 2013-4 & 289 & $63 \%$ & S/. $1,169,260$ & $72 \%$ & 98 & $21 \%$ & S/. 239,366 & $15 \%$ & 68 & $15 \%$ & S/. 187,523 & $12 \%$ & 2 & $0.4 \%$ & S/ 33,978 & $2 \%$ & 457 & $5.2 \%$ & S/. 1,630,127 & $6.4 \%$ \\
\hline 13 & $2014-1$ & 352 & $62 \%$ & S/. $1,257,131$ & $55 \%$ & 118 & $21 \%$ & S/. 537,862 & $24 \%$ & 93 & $16 \%$ & S/. 479,370 & $21 \%$ & 2 & $0.4 \%$ & S/. 4,279 & $0.2 \%$ & 565 & $6.5 \%$ & S/. 2,278,642 & $\mathbf{9 . 0} \%$ \\
\hline 14 & 2014-2 & 190 & $50 \%$ & S/. 721,188 & $51 \%$ & 96 & $25 \%$ & S/. 319,104 & $22 \%$ & 97 & $25 \%$ & S/. 378,698 & $27 \%$ & 0 & $0 \%$ & S/. 0.00 & $0 \%$ & 383 & $4.4 \%$ & S/. 1,418,990 & $5.6 \%$ \\
\hline 15 & $2014-3$ & 317 & $59 \%$ & S/ 1,200,506 & $59 \%$ & 122 & $23 \%$ & S/. 428,285 & $21 \%$ & 102 & $19 \%$ & S/. 410,020 & $20 \%$ & 0 & $0 \%$ & S/. 0.00 & $0 \%$ & 541 & $6.2 \%$ & S/ 2,038,811 & $8.0 \%$ \\
\hline 16 & 2014-4 & 403 & $71 \%$ & S/. $1,376,468$ & $68 \%$ & 107 & $19 \%$ & S/. 337,763 & $17 \%$ & 54 & $10 \%$ & S/. 324,698 & $16 \%$ & 0 & $0 \%$ & S/. 0.00 & $0 \%$ & 564 & $6.4 \%$ & S/. 2,038,929 & $8.0 \%$ \\
\hline 17 & $2015-1$ & 317 & $61 \%$ & S/. 943,280 & $56 \%$ & 110 & $21 \%$ & S/. 310,139 & $18 \%$ & 95 & $18 \%$ & S/. 441,410 & $26 \%$ & 0 & $0 \%$ & $\mathrm{~S} / .0 .00$ & $0 \%$ & 522 & $6.0 \%$ & S/. 1,694,829 & $6.7 \%$ \\
\hline 18 & 2015-2 & 391 & $68 \%$ & S/. 1,290,741 & $60 \%$ & 120 & $21 \%$ & S/. 576,010 & $27 \%$ & 65 & $11 \%$ & S/. 289,684 & $13 \%$ & 0 & $0 \%$ & S/. 0.00 & $0 \%$ & 576 & $6.6 \%$ & S/. 2,156,435 & $8.5 \%$ \\
\hline & & & & & & & & & & & & & & & & & & 8752 & $100 \%$ & S/. 25,425,932 & $100 \%$ \\
\hline
\end{tabular}


La tabla 4 presenta las cuentas por cobrar en los próximos trimestres de los años 2015, 2016 y 2017; organizadas según tipos de cartera. En la cobranza inicial correspondiente a la cartera atrasada, habrá un incremento considerable en los trimestres de los años 2015, 2016 y 2017; las operaciones en el tercer trimestre del año 2015 son de 380 comprobantes, con un monto de S/.1.370,344 equivalente a un 59\%; en el cuarto trimestre del 2015 incrementa a 394 operaciones con un importe de S/. 1,431,787, en el primer trimestre del 2016 se incrementa a 408 operaciones con un importe de S/. 1,493,229 equivalente a 59\%; en el segundo trimestre del 2016 incrementa a 422 operaciones con un importe de S/1,554,671 equivalente a un 59\%, en el tercer trimestre del 2016 se incrementa las operaciones a 436 comprobantes con un importe de S/. 1,616,114 equivalente a 59\%; en el cuarto trimestre del 2016 se incrementan a 450 comprobantes S/. 1,677,556 equivalente a 60\%; en el primer trimestre del 2017 las operaciones se incrementan a 464 comprobantes con un importe de S/. 1,738,998 equivalente a 60\%; en el segundo trimestre del 2017 se incrementan las operaciones en 478 comprobantes con importe de S/. 1,800,441 equivalente a 60\%; en el tercer trimestre de 2017 se incrementan a 498 comprobantes y con un importe S/. 1,861,883 equivalente a 60\%; en cuarto trimestre de 2017 se incrementa las operaciones en 506 comprobantes con un importe de S/. 1,923,325 equivalente a 60\%, los cuales indican que las cobranzas iniciales se incrementaran satisfactoriamente.

En cuanto a la cobranza vencida habrá una disminución considerablemente en los años 2015, 2016 y 2017 ; en el tercer trimestre de 2015 disminuirá a 78 comprobantes vencidas con importe de S/. 470,952; en el cuarto trimestre de 2015 es de 74 comprobantes y con importe de S/. 486,048; en el primer trimestre de 2016 es de 70 comprobantes con un importe de S/. 501,145; en el segundo trimestre de 2016 los comprobantes vencidos disminuyen a 66 y con un importe de S/. 516,241; en el tercer trimestre de 2016 disminuirá a 61 comprobantes vencidas con un importe S/. 531,338; en cuarto trimestre de 2016 disminuirá a 57 comprobantes y con un importe S/. 546,435; en el primer trimestre de 2017 disminuirá a 53 comprobantes vencidas con un importe de S/. 561,531; en el segundo trimestre de 2017 los comprobantes disminuirán a 48 con un importe S/. 576,628; el tercer trimestre de 2017 disminuirá a 44 comprobantes vencidas con importe S/. 591,724; el cuarto trimestre de 2017 disminuirá a 40 comprobantes vencidas con un importe S/. 606,821.

La tendencia de la cartera pesada correspondiente la cobranza con problemas potenciales, en los próximos trimestres de los años 2015, 2016 y 2017, organizadas según tipos de cartera.

Las operaciones en el tercer trimestre del año 2015 serán 94 comprobantes, con un importe de S/.487,545; en el cuarto trimestre del 2015 disminuirá a 92 comprobantes con un importe de S/.508,389; en el primer trimestre del año 2016 serán 91 comprobantes con un importe de S/.529,233; en el segundo trimestre del año 2016 disminuirá a 89 comprobantes con un importe de S/.550,077; en el tercer trimestre del año 2016 disminuirá a 87 comprobantes con un importe de S/.570,921; en el cuarto trimestre del año 2016 disminuirá a 86 comprobantes con un importe de S/.591,765, en el primer trimestre del año 2017 serán 84 comprobantes con un importe de S/.612;609; en el segundo trimestre del año 2017 disminuirá a 83 comprobantes con un importe de S/.633,45; en el tercer trimestre del año 2017 disminuirá a 81 comprobantes con un importe de S/.654,297; en el cuarto trimestre del año 2017 disminuirá a 80 comprobantes con un importe de S/.675,140.

Por último, la cartera de alto riesgo, en los próximos trimestres de los años 2015, 2016 y 2017 será nula entendiéndose.

Tabla 4

Tendencia de comprobantes de pago de operaciones de ventas al crédito canceladas, según tipo de cartera de la empresa Alpe Corporación S.A. en el periodo 2011 al 2017.

\begin{tabular}{|c|c|c|c|c|c|c|c|c|c|c|c|c|c|c|c|c|c|c|c|c|c|}
\hline \multirow{3}{*}{$\mathbf{N}^{\circ}$} & \multirow{3}{*}{ Año } & \multicolumn{8}{|c|}{ Cartera atrasada } & \multicolumn{4}{|c|}{ Carteras Pesadas } & \multicolumn{8}{|c|}{ Carteras de alto riesgo } \\
\hline & & \multicolumn{4}{|c|}{ Cobranza Inicial (normal) } & \multicolumn{4}{|c|}{ Cobranza vencida } & \multicolumn{4}{|c|}{ Con problemas potenciales. } & \multicolumn{4}{|c|}{ Castigo de cuentas incobrables } & \multicolumn{4}{|c|}{ TOTAL } \\
\hline & & $\begin{array}{c}\text { Cantidad } \\
\text { Factura } \\
\text { Emitido }\end{array}$ & $\%$ & Monto Facturado & $\%$ & $\begin{array}{l}\text { Cantidad } \\
\text { Factura } \\
\text { Emitido }\end{array}$ & $\%$ & Monto Facturado & $\%$ & $\begin{array}{l}\text { Cantidad } \\
\text { Factura } \\
\text { Emitido }\end{array}$ & $\%$ & Monto Facturado & $\%$ & $\begin{array}{l}\text { Cantidad } \\
\text { Factura } \\
\text { Emitido }\end{array}$ & $\%$ & $\begin{array}{c}\text { Monto } \\
\text { Facturado }\end{array}$ & $\%$ & $\begin{array}{c}\text { Cantidad } \\
\text { Factura } \\
\text { Emitido }\end{array}$ & $\%$ & Monto Facturado & $\%$ \\
\hline 19 & $2015-3$ & 380 & $69 \%$ & S/.1,370,344 & $59 \%$ & 78 & $14 \%$ & S/.470,952 & $20 \%$ & 94 & $17 \%$ & S/.487,545 & $21 \%$ & 0 & $0 \%$ & $\mathrm{~S} / .0$ & $0 \%$ & 553 & $9 \%$ & S/. 2,328,841 & $8 \%$ \\
\hline 20 & $2015-4$ & 394 & $70 \%$ & S/.1,431,787 & $59 \%$ & 74 & $13 \%$ & S/.486,048 & $20 \%$ & 92 & $16 \%$ & S/.508,389 & $21 \%$ & 0 & $0 \%$ & $\mathrm{~S} / .0$ & $0 \%$ & 561 & $10 \%$ & S/. 2,426,223 & $9 \%$ \\
\hline 21 & 2016-1 & 408 & $72 \%$ & $\mathrm{~S} / .1,493,229$ & $59 \%$ & 70 & $12 \%$ & S/.501,145 & $20 \%$ & 91 & $16 \%$ & S/.529,233 & $21 \%$ & 0 & $0 \%$ & $\mathrm{~S} / .0$ & $0 \%$ & 569 & $10 \%$ & S/. 2,523,606 & $9 \%$ \\
\hline 22 & 2016-2 & 422 & $73 \%$ & S/.1,554,671 & $59 \%$ & 66 & $11 \%$ & S/.516,241 & $20 \%$ & 89 & $15 \%$ & S/.550,077 & $21 \%$ & 0 & $0 \%$ & $\mathrm{~S} / .0$ & $0 \%$ & 577 & $10 \%$ & S/. 2,620,989 & $9 \%$ \\
\hline 23 & 2016-3 & 436 & $75 \%$ & S/.1,616,114 & $59 \%$ & 61 & $10 \%$ & S/.531,338 & $20 \%$ & 87 & $15 \%$ & S/.570,921 & $21 \%$ & 0 & $0 \%$ & $\mathrm{~S} / .0$ & $0 \%$ & 585 & $10 \%$ & S/. 2,718,372 & $10 \%$ \\
\hline 24 & 2016-4 & 450 & $76 \%$ & S/.1,677,556 & $60 \%$ & 57 & $10 \%$ & S/.546,435 & $19 \%$ & 86 & $14 \%$ & S/.591,765 & $21 \%$ & 0 & $0 \%$ & $\mathrm{~S} / .0$ & $0 \%$ & 593 & $10 \%$ & S/. 2,815,755 & $10 \%$ \\
\hline 25 & $2017-1$ & 464 & $77 \%$ & S/.1,738,998 & $60 \%$ & 53 & $9 \%$ & S/.561,531 & $19 \%$ & 84 & $14 \%$ & S/.612,609 & $21 \%$ & 0 & $0 \%$ & $\mathrm{~S} / .0$ & $0 \%$ & 601 & $10 \%$ & S/. 2,913,138 & $11 \%$ \\
\hline 26 & $2017-2$ & 478 & $78 \%$ & S/.1,800,441 & $60 \%$ & 48 & $8 \%$ & S/.576,628 & $19 \%$ & 83 & $14 \%$ & S/.633,453 & $21 \%$ & 0 & $0 \%$ & $\mathrm{~S} / .0$ & $0 \%$ & 609 & $10 \%$ & $\mathrm{~S} / . \mathbf{3 , 0 1 0 , 5 2 1}$ & $11 \%$ \\
\hline 27 & $2017-3$ & 492 & $80 \%$ & $\mathrm{~S} / .1,861,883$ & $60 \%$ & 44 & $7 \%$ & S/.591,724 & $19 \%$ & 81 & $13 \%$ & S/.654,297 & $21 \%$ & 0 & $0 \%$ & $\mathrm{~S} / .0$ & $0 \%$ & 618 & $10 \%$ & S/. 3,107,904 & $11 \%$ \\
\hline 28 & $2017-4$ & 506 & $81 \%$ & S/.1,923,325 & $60 \%$ & 40 & $6 \%$ & S/.606,821 & $19 \%$ & 80 & $13 \%$ & S/.675,140 & $21 \%$ & 0 & $0 \%$ & $\mathrm{~S} / .0$ & $0 \%$ & 626 & $11 \%$ & S/. 3,205,286 & $12 \%$ \\
\hline & & & & & & & & & & & & & & & & & & 5892 & $100 \%$ & S/. 27,670,635 & $100 \%$ \\
\hline
\end{tabular}


Tendencia de Ratios de actividad de la empresa Alpe Corporación S.A. en el periodo 2011 al 2017

A continuación, se presenta el análisis de los ratios de actividad para determinar la frecuencia de recuperación de cuentas por cobrar y la rotación trimestral de la empresa Alpe Corporación S.A. durante el periodo 2011 al 2017 teniendo como datos históricos disponibles del 2011 a junio de 2015. El Modelo de regresión lineal está presentado por los coeficientes de Beta que son significativos por lo que el valor P o sig < 0,05; los coeficientes del modelo de regresión, $\boldsymbol{\beta}_{0}$ (constante) y $\boldsymbol{\beta}_{\mathbf{1}}$ (dependiente), permiten formular el modelo para poder pronosticar las rotaciones trimestrales, que se muestra a continuación:

En la figura 1, salvo un punto atípico, se aprecia que hay relación lineal inversamente proporcional entre las variables. Es decir, a medida que la rotación de cuentas por cobrar aumenta, la rotación trimestral disminuye. Lo que significa que la empresa Alpe Corporación S.A. es eficiente para generar fondos de manera efectiva administrando de forma adecuada los servicios prestados proyectados a los años que falten de julio del 2015 hasta el 2017.

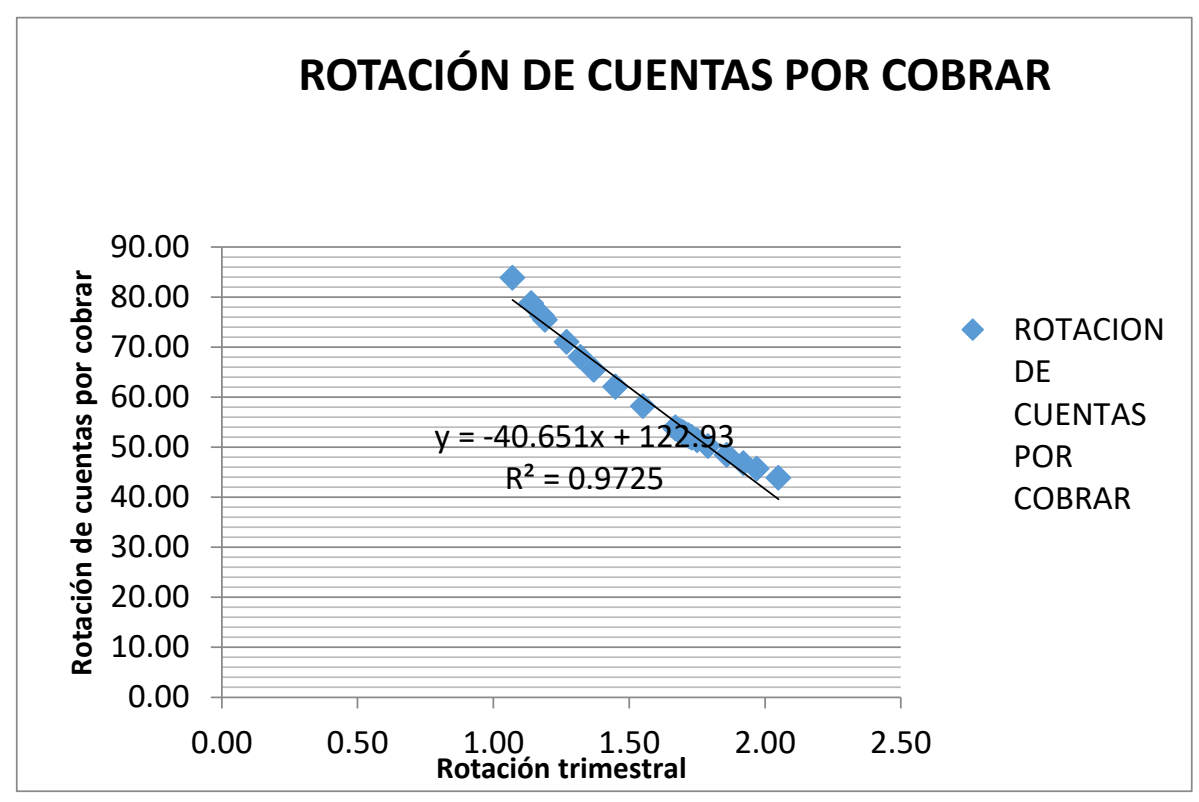

Figura 1. Diagrama de dispersión de la Rotación de cuentas por cobrar y rotación trimestral

En la tabla 5 se muestra el análisis del modelo ANOVA y el nivel de significancia y correlación entre la rotación de cuentas por cobrar y rotación trimestral del periodo 2011 al 2017 de la empresa Alpe Corporación S.A.

Asimismo, en la tabla 5 la relación entre las variables es alta en el coeficiente de correlación $\mathrm{r}=$,986 y el coeficiente de determinación r2 = ,973. Es decir, la variación de la rotación trimestral explicada por el modelo de regresión lineal, por lo cual, se puede predecir la rotación trimestral a partir de la Rotación de cuentas por cobrar al 98.6\%. A esto el valor $\mathrm{P}$ o sig $=, 000<, 05(\alpha)$ indica que el modelo propuesto de regresión es significativo.

Luego, teniendo en cuenta que el nivel óptimo de rotación de las cuentas por cobrar es de 6 a 12 veces al año y de 60 a 30 días de periodo de cobro; para fines de este estudio se considera un periodo desde el año 2011 al primer semestre del 2015 de acuerdo a los datos históricos disponibles. Es así que el nivel óptimo de rotación de cuentas por cobrar es de 1.5 a 3 veces por trimestre y de 60 a 30 días de periodo de cobro normal.

Tabla 5

Resumen del modelo, ANOVA y los coeficientes de Rotación de cuentas por cobrar y rotación trimestral.

\begin{tabular}{|c|c|c|c|c|c|c|}
\hline \multicolumn{3}{|c|}{ Resumen del modelo $^{\mathrm{b}}$} & \multirow{3}{*}{$\begin{array}{l}\text { ANOVA }^{\mathrm{a}} \\
\text { Sig. }\end{array}$} & \multicolumn{3}{|c|}{ Coeficientes $^{\mathrm{a}}$} \\
\hline \multirow[t]{2}{*}{ Modelo } & \multirow[t]{2}{*}{$\mathrm{R}$} & \multirow[t]{2}{*}{$\mathrm{R}$ cuadrado } & & & $\begin{array}{l}\text { Coeficientes no } \\
\text { estandarizados }\end{array}$ & \multirow[t]{2}{*}{ Sig. } \\
\hline & & & & & $\mathrm{B}$ & \\
\hline & & & & (Constante) & 122,926 & 000 \\
\hline 1 &, $986^{\mathrm{a}}$ & ,973 &, $000^{\mathrm{b}}$ & $\begin{array}{l}\text { Rotación de } \\
\text { cuentas por } \\
\text { cobrar }\end{array}$ & -40.651 & ,000 \\
\hline
\end{tabular}

a. Predictores: (Constante), Rotación de cuentas por cobrar

En la tabla 6 se observa que la empresa convierte en efectivo sus cuentas por cobrar en 53.73 días o rotan 1.67 veces en el tercer trimestre del año 2015; y en 53.47 días o rotan 1.68 veces durante el cuarto trimestre del año 2015. 
La empresa convierte en efectivo sus cuentas por cobrar en 53.23 días o rotan 1.69 veces en el primer trimestre del 2016; en 53.01 días o rotan en 1.70 veces durante el segundo trimestre del 2016, en 52.80 días o rotan 1.70 veces durante el tercer trimestre del 2016, en 52.60 días o rotan 1.71 veces durante el cuarto trimestre del 2016.

La empresa convierte en efectivo sus cuentas por cobrar en 52.42 días o rotan 1.72 veces durante el primer trimestre del 2017, en 52.25 días o rotan 1.72 veces durante el segundo trimestre del 2016; en 52.09 días o rotan en 1.73 veces en el tercer trimestre del 2017; en 51.94 días o rotan 1.73 veces durante el cuarto trimestre del 2017, significa que a medida que la rotación de cuentas por cobrar disminuye, la rotación trimestral aumenta.

Tabla 6.

Estimación de rotación de cuentas por cobrar para los siguientes años y estimación de rotación Trimestral.

\begin{tabular}{ccc}
\hline Trimestral & Estimación de rotación de cuentas por cobrar & Estimación de rotación Trimestral \\
\hline $2015 \_3$ & 53.73 & 1.67 \\
$2015 \_4$ & 53.47 & 1.68 \\
$2016-1$ & 53.23 & 1.69 \\
$2016-2$ & 53.01 & 1.70 \\
$2016-3$ & 52.80 & 1.70 \\
$2016-4$ & 52.60 & 1.71 \\
$2017-1$ & 52.42 & 1.72 \\
$2017-2$ & 52.25 & 1.72 \\
$2017-3$ & 52.09 & 1.73 \\
$2017-4$ & 51.94 & 1.73 \\
\hline
\end{tabular}

\section{Discusión}

En cuanto a las cuentas por cobrar según el tipo de carteras, en los trimestres del año 2011. Se observa que la cobranza vencida de la cartera atrasada, registra la mayor cantidad de comprobantes con retraso y la cartera de alto riesgo registra un $25 \%$ de comprobantes con retraso, en tal sentido el sistema de cobranzas de la empresa debería haber mejorado la estrategia de gestión al otorgar los créditos a sus clientes. Sin embargo, en los trimestres de los años 2012, 2013, 2014 y los dos primeros trimestres del 2015, se observa que los comprobantes de la cobranza inicial se han incrementado.

Así mismo, se estima que, en los años 2015, 2016 y 2017 la recuperación de las cuentas por cobrar se incrementará significativamente y disminuirán las cobranzas vencidas. Lo cual indica que la política de gestión de cobranzas de la empresa ha mejorado considerablemente. Al respecto Noriega (2011) manifestó que es necesario aplicar políticas, procedimientos y períodos de crédito adecuados al giro del negocio, que proyecte beneficios económicos a las empresas; además de aplicar medidas de control y seguimiento a dichas políticas conforme las necesidades y evolución del negocio. Se describen, también, herramientas de análisis de cartera con el fin de que los mismos contribuyan a administrar las cuentas por cobrar, de forma clara, sencilla, responsable y eficiente.

En cuanto a la estimación de rotación de cuentas por cobrar, para los siguientes años y estimación de rotación trimestral, se observa que la rotación de cuentas por cobrar, así como la rotación trimestral se encuentra en el nivel óptimo. Por lo cual se concluye que la capacidad gerencial de la empresa es eficiente para generar fondos de manera efectiva administrando de forma adecuada los servicios prestados en el 2015, 2016 y 2017. Esto corrobora lo señalado por Lizárraga (2010). Sin una buena política de créditos no será posible que una empresa alcance niveles de cuentas por cobrar razonables que favorezcan la rotación de las cuentas por cobrar y la asignación correcta del crédito. Una gestión eficiente de las cuentas por cobrar significa también estar integrada a una plataforma tecnológica bancaria, para poder acceder al financiamiento.

\section{Conclusiones}

Primera conclusión: La tendencia de las cuentas por cobrar y los ratios de actividad evidencia la eficiencia en la empresa Alpe Corporación S.A. en el periodo 2011 al 2017. Por lo cual, la empresa convierte en efectivo sus cuentas por cobrar en la medida que los días aumentan y las veces de cobro disminuye en cada trimestre de cada año. Segunda Conclusión: La cobranza inicial, 
correspondiente a la cartera atrasada, se incrementará significativamente hasta el 2017 y se obtendrá un $81 \%$ en la cantidad de comprobantes emitidas, y un $60 \%$ en la recuperación de las cuentas por cobrar. Mientras que la cobranza vencida disminuirá significativamente hasta el 2017 y se obtendrá un 6\% en la cantidad de comprobantes emitidas y un 19\% en la recuperación de las cuentas por cobrar. Tercera Conclusión: La Cartera pesada de la cobranza con problemas potenciales disminuirá hasta el 2017, en un $13 \%$ en la cantidad de comprobantes emitidas y un $21 \%$ en la recuperación de las cuentas por cobrar. Cuarta Conclusión: Las carteras de alto riesgo con castigos de cuentas incobrables disminuirán significativamente hasta el 2017 en un $0 \%$. Quinta Conclusión: La rotación de cuentas por cobrar, así como la rotación trimestral, se encuentra en el nivel óptimo. Lo que significa que la capacidad gerencial de la empresa es eficiente para generar fondos de manera efectiva administrando de forma adecuada los servicios prestados proyectados a los trimestres que falten del tercer y cuarto trimestre del 2015, 2016 hasta el 2017.

\section{Recomendaciones}

Analizar la capacidad de endeudamiento de cada cliente, estableciendo límites de crédito a fin de que la empresa pueda recuperar las cuentas por cobrar en plazos previstos y así tener una mayor rotación de estas.

Así mismo, la empresa deberá restablecer sus políticas de crédito y revisar periódicamente para cambiar, modificar, adaptar o actualizar conforme a las necesidades de la empresa.

Se recomienda realizar un análisis de antigüedad de la cartera, así como utilizar el análisis de modelo de regresión, lo cual permitirá a la gerencia de la empresa conocer la situación real de su cartera, así como el grado de efectividad que tiene el área de gestión de cuentas por cobrar.

Capacitar a todo personal que esté involucrado en la administración de créditos, cobros y ventas, puesto que las tres áreas están interrelacionadas y son necesarias para una administración óptima de cuentas por cobrar. Para un mejor control de las cuentas por cobrar, se deberá realizar auditorías trimestralmente.

\section{Referencias}

Aching Guzman, C. (2006) Ratios financieros y matemáticas de la mercadotécnia. Diplomado Esan, Serie Mypes pág, $15-31$. Recuperado de https://puntodevistaypropuesta.files.wordpress.com/2015/03/ratios-financieros-y-matematicas-de-lamercadotecnia.pdf.

Aguilar Pinedo, V. H. (2013). Gestión de cuentas por cobrar y su incidencia en la liquidéz de la empresa contratista Corporación Petrolera SAC-Año 2012. Tesis de licenciatura. Universidad San Martín de Porres.

Farro Espino, C. D. M., \& Carrasco Odar, M. D. P. (2014). Evaluación del control interno a las cuentas por cobrar de la Empresa de Transportes y Servicios Vanina EIRL. Para mejorar la eficiencia y gestión durante el período 2012. Tesis de licenciatura. Universidad Católica Santo Toribio de Mogrovejo.

Hernandez Gonzalez, J. M. (2013). Metodo Predictivo en Prezi. Retrieved 28 Agosto, 2013, from https://prezi.com/ozyanscpar3q/metodo-predictivo/.

Noriega Castro J. V. (2011) Administración de cuentas por cobrar-un enfoque para la toma de decisiones en la industria maquiladora de prendas de vestir en Guatemala. Tesis de maestría. Universidad de San Carlos de Guatemala.

Lizárraga Vergaray, K. E. (2010). Gestión de las cuentas por cobrar en la administración del capital de trabajo de las empresas de fabricación de plástico del distrito de Ate. Tesis de licenciatura. Universidad de San Martín de Porres. 